\title{
Original
}

\section{The Relationship between Jaw Function and Cognitive Function in the Elderly Population}

\author{
Kei Suzuki ${ }^{1)}$, Nahoko Kato-Kogoe ${ }^{1)}$, Kazuya Inoue ${ }^{1)}$, Michi Omori ${ }^{1)}$, Naoko Imagawa ${ }^{1)}$, Hiroyuki Nakano ${ }^{1)}$, Yoshifumi Suwa ${ }^{1)}$, \\ Kayoko Yamamoto $^{2)}$, Satoyo Ikehara ${ }^{3)}$, Hironobu Kakihana ${ }^{3)}$, Akemi Nitta ${ }^{3)}$, Mizuho Nagata ${ }^{3)}$, Kuniyasu Kamiya ${ }^{3)}$, \\ Tetsuya Togashi ${ }^{4)}$, Jyunko Tamaki ${ }^{3)}$, Masaaki Hoshiga ${ }^{5}$ and Takaaki Ueno ${ }^{1)}$ \\ 1) Department of Oral Surgery, Osaka Medical College, Osaka, Japan \\ 2) Department of Oral Biological \& Medical Sciences, Faculty of Dentistry, The University of British Columbia, Vancouver, Canada. \\ 3) Department of Hygiene and Public Health, Osaka Medical College, Osaka, Japan \\ 4) Department of Neuropsychiatry, Osaka Medical College, Osaka, Japan \\ ${ }^{5)}$ Department of Cardiology, Osaka Medical College, Osaka, Japan \\ (Accepted for publication, April 12, 2020)
}

\begin{abstract}
Tooth loss is one of the most important factors in preventing the development of dementia in aging societies. Although numerous studies focusing on this relationship have been conducted, few studies have investigated the relationship between oral function and the development of dementia. Therefore, in the present study, we examined the relationship between oral and cognitive functions in the elderly population. This study included 108 residents of Takatsuki City aged 65 years or older. We analyzed jaw function based on each individual's tooth loss and periodontal condition. In addition, we used the Japanese version of the Mini-Mental State Examination and Trail Making Test (TMT) A/B to assess subjects' cognitive function. Spearman's rank-order correlation coefficient was used in the statistical analysis. There was a significant negative correlation between the number of teeth, maximum occlusal force, and scores on the TMT A and B times. There was also a significant negative correlation between the tongue pressure and the TMT A time. The present study's results suggest that dental and hard tissues should be actively reconstructed in the elderly population to help prevent the development of dementia.
\end{abstract}

Key words: Maximum occlusal force, Tongue pressure, Gummy score, TMT-A, TMT-B, Dementia

\section{Introduction}

In aging societies such as Japan, the number of teeth one has is one the most important factor in the development of dementia. Several risk factors for dementia have been reported. In a longitudinal study, loss of multiple teeth was found to be associated with a higher risk of morbidity and an increased incidence of dementia ${ }^{1}$. Therefore, tooth loss is considered to be a major risk factor for the development of dementia ${ }^{2}$. Although numerous studies have investigated the association between tooth loss and cognitive function, few studies have investigated the relationship between oral and cognitive functions ${ }^{3)}$.

In the present study, we examined the relationship between oral and cognitive functions in the elderly population with the aim of preventing the development of dementia.

\section{Materials and Methods}

\section{Ethical considerations}

This study was approved by the Ethics Committee of Osaka Medical College (approval number 2309), and it conforms to accepted ethical standards formulated in the Helsinki Declaration of 1964 (revised in 2000). All participants provided informed consent for the oral examinations and function assessments.

Correspondence to: Dr. Kei Suzuki, Department of Oral Surgery, Osaka Medical College, 2-7 Daigaku-machi, Takatsuki City, Osaka, 569-8686, Japan; Tel: +81-72-683-1221; Fax: +81-72-684-6833; E-mail: ora096@osaka-med. ac.jp

\section{Participants}

Subjects of the present study were 53 men and 55 women aged 65 years or older living in Takatsuki City (108 subjects). They underwent the following examination in November 2017.

\section{Examination items}

All participants took part in an individual interview, followed by an oral examination performed by dentists. The following items were assessed.

Oral function

- Number of teeth

-Maximum occlusal force: Maximum occlusal force was measured once for each side using a simple occlusal force-measuring device (Occlusal Force Meter GM10; Nagano Keiki Co., Ltd., Tokyo, Japan) to determine the maximum value for each subject.

-Masticatory performance : The gummy jelly score was used to assess mastication ability. To obtain gummy jelly scores, each subject chewed one test gummy jelly (UHA Mikakuto Co., Ltd., Osaka, Japan) as the chewing sample 30 times, and then spit the comminuted pieces into a gauze cloth. The pieces were transferred to a transparent petri dish and used as samples for visual inspection; two evaluators visually compared the size of the pieces in the same sample on a scale of 1 to 10 in order to obtain the gummy score If the scores of the two evaluators differed, the higher score was used $^{4,5)}$.

-Tongue pressure: Tongue pressure was measured using a tongue 
J.Hard Tissue Biology Vol. 29(3): 169-172, 2020

Table 1. Summary of oral conditions and cognitive function

\begin{tabular}{cc}
\hline Examination item & $\begin{array}{c}\text { Median (min-max) } \\
\text { Mean } \pm \text { S.D. }\end{array}$ \\
\hline $\begin{array}{c}\text { Number of teeth (number) } \\
\text { Maximum occlusal force }(\mathrm{N}) \\
\text { Masticatory performance } \\
\text { (Gummy jelly score) }\end{array}$ & $23.0(0-28)$ \\
Tongue pressure (kPa) & $5(0-9)$ \\
TMT A time (s) & $26.3 \pm 9.0$ \\
TMT B time (s) & $46.5(39.0-53.7)$ \\
\hline
\end{tabular}

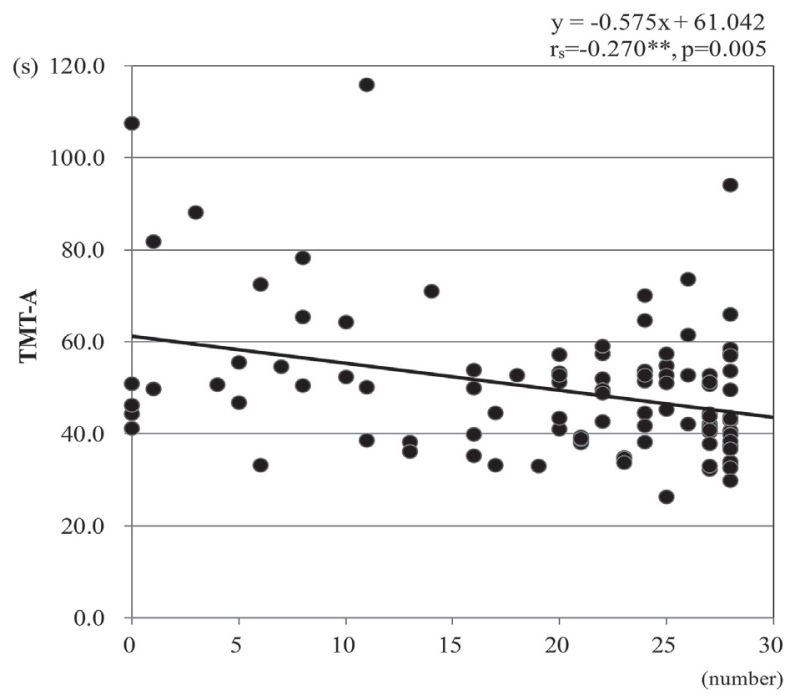

Number of teeth

Figure 1. The correlation between number of teeth and TMT-A. We conducted statistical analysis with Spearman's rank-order correlation coefficient. $\mathrm{r}_{\mathrm{s}}$ : Spearman's rank correlation coefficient, $* *: \mathrm{p}<0.01$. There is a negative correlation between number of teeth and TMT-A.

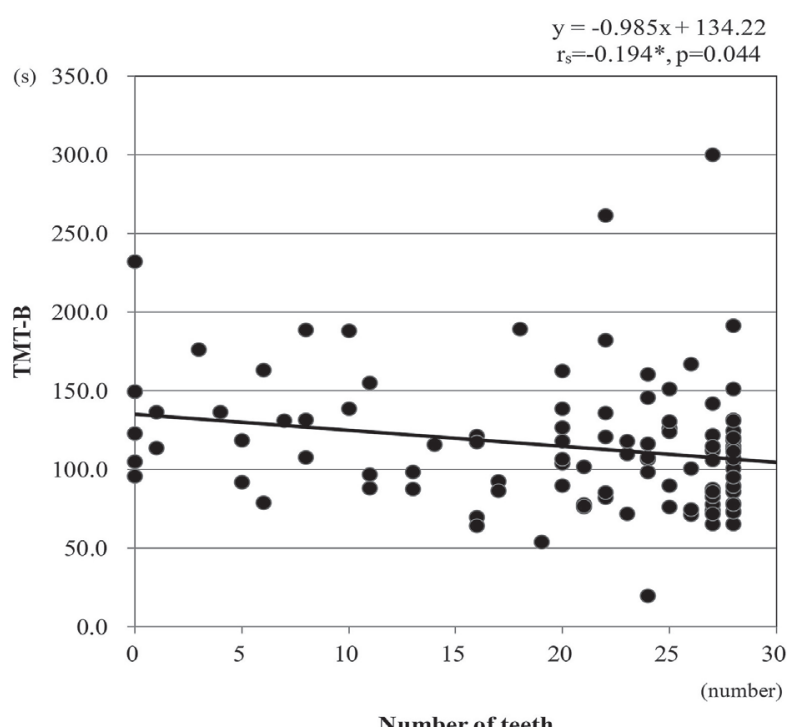

Figure 2. The correlation between number of teeth and TMT-B. We conducted statistical analysis with Spearman's rank-order correlation coefficient. $r_{s}$ : Spearman's rank correlation coefficient, *: $p<0.05$. There is a negative correlation between number of teeth and TMT-B.

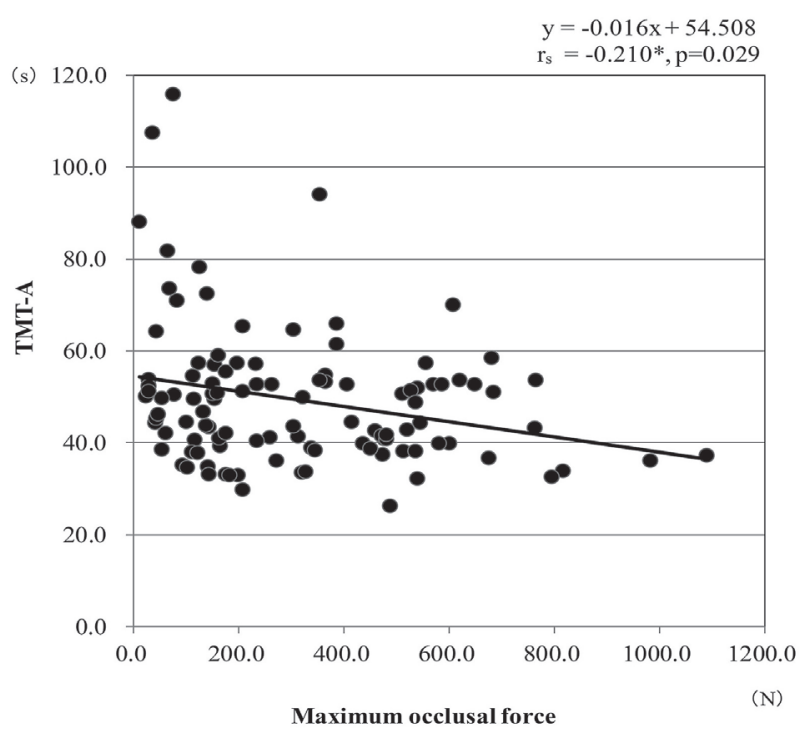

Figure 3. The correlation between maximum occlusal force and TMT-A. We conducted statistical analysis with Spearman's rank-order correlation coefficient. $\mathrm{r}_{\mathrm{s}}$ : Spearman's rank correlation coefficient, *: $\mathrm{p}<0.05$. There is a negative correlation between maximum occlusal force and TMT-A.

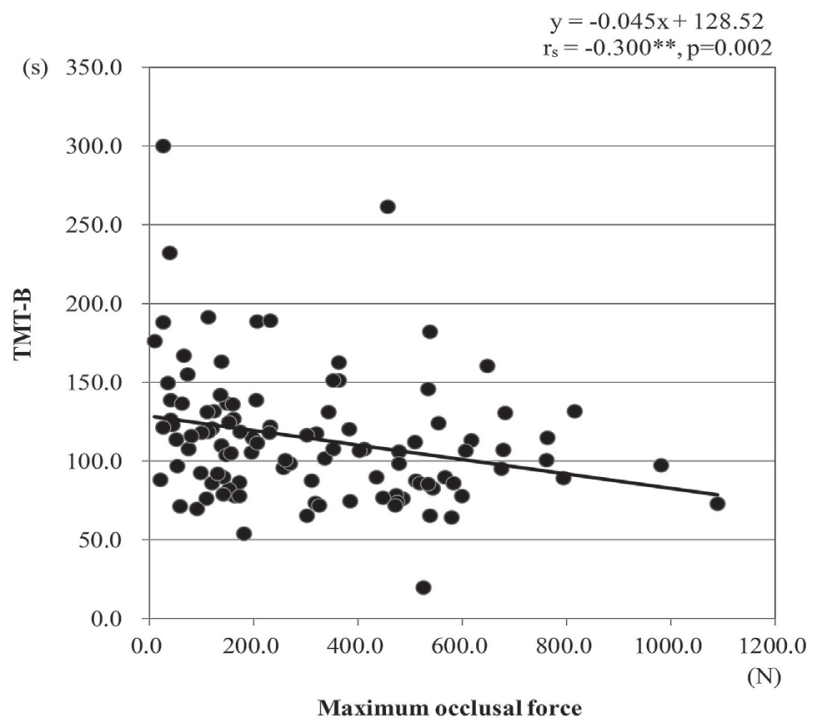

Figure 4. The correlation between maximum occlusal force and TMT-B. We conducted statistical analysis with Spearman's rank-order correlation coefficient. $\mathrm{r}_{\mathrm{s}}$ : Spearman's rank correlation coefficient, ${ }^{* *}: \mathrm{p}<0.01$. There is a negative correlation between maximum occlusal force and TMT-B.

pressure-measuring device (JMS tongue pressure measurement device; Morita Corporation, Tokyo, Japan).

Cognitive function

- Trail Making Test (TMT) A: Numbers from 1 to 25 were randomly arranged on a sheet of paper. The participants connected the numbers in consecutive order in a single line. Then time required to reach the last number (number 25) was measured.

•TMT B: Numbers from 1 to 13 and letters from “あ” to “し” were randomly arranged on a sheet of paper. The participants alternately draws a line between numbers and kana such as $1 \rightarrow$ あ $\rightarrow 2 \rightarrow$ い $\rightarrow 3 \ldots$ as quickly as possible. Then the time required to reach the 
Kei Suzuki et al.: Oral and Cognitive Function in the Elderly Population

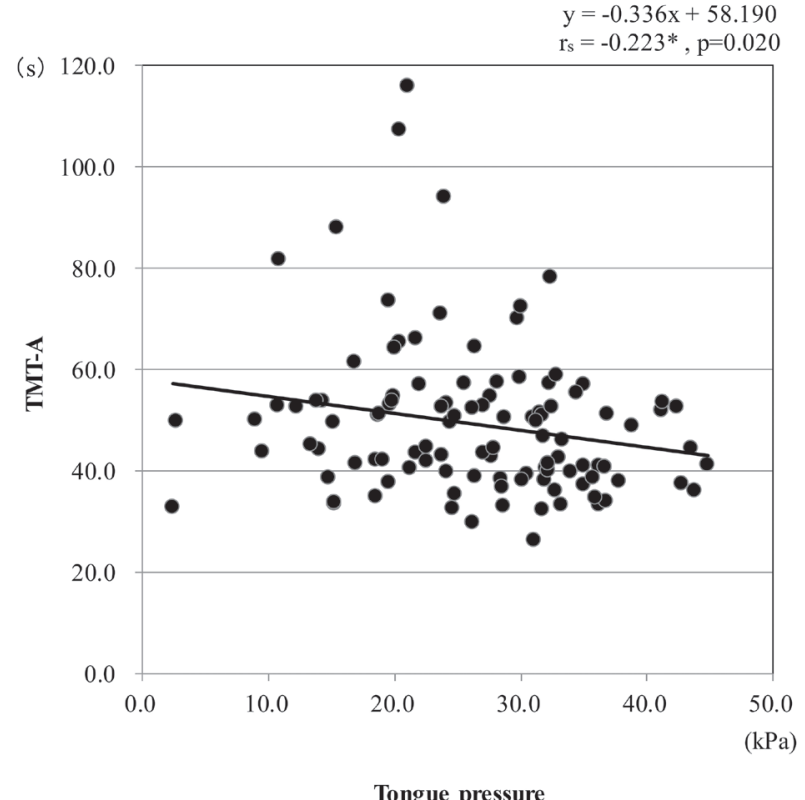

Figure 5. The correlation between tongue pressure and TMT-A. We conducted statistical analysis with Spearman's rank-order correlation coefficient. rs: Spearman's rank correlation coefficient, *: $\mathrm{p}<0.05$. There is a negative correlation between tongue pressure and TMT-A.

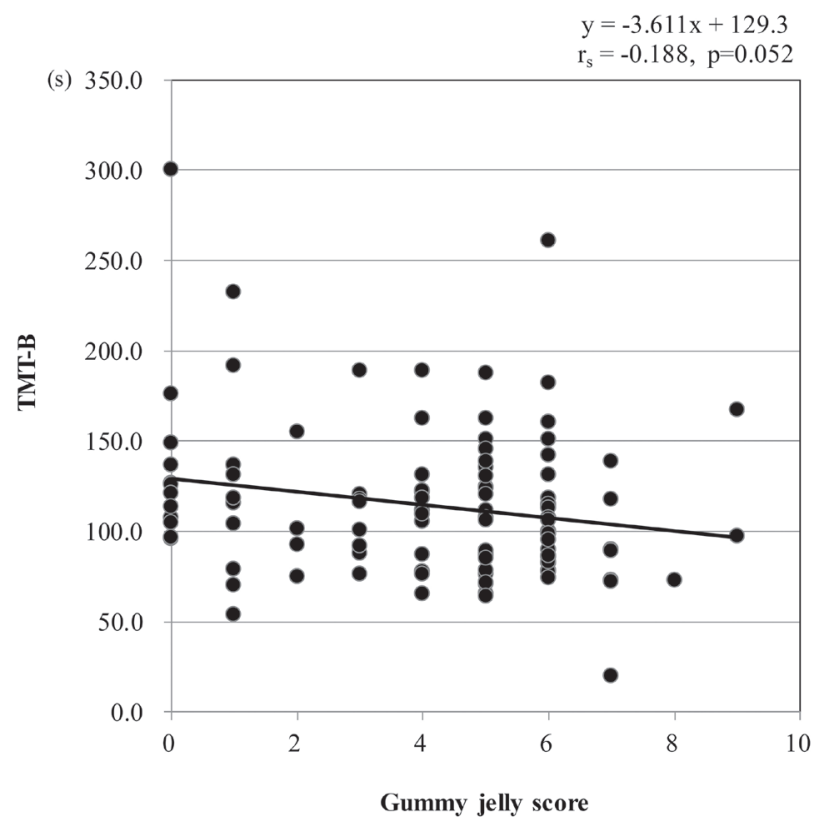

Figure 6. The correlation between Masticatory performance and TMT-B. We conducted statistical analysis with Spearman's rank-order correlation coefficient. $r_{\mathrm{s}}$ : Spearman's rank correlation coefficient. There is a negative correlation between Masticatory performance and TMT-B.

last letter (“し”) was measured.

-Mini-Mental State Examination (MMSE)-Japanese version (MMSE-J): The MMSE-J was used to test for dementia (memorization faculty, faculty of orientation, calculating ability, etc.).

\section{Statistical analysis}

The correlation coefficients for age, number of teeth, maximum oc- clusal force, masticatory performance, tongue pressure, TMT A time, TMT B time, and MMSE-J score were calculated. We conducted statistical analysis using Spearman's rank-order correlative coefficient and multiple linear regression analyses to evaluate the associations between indicators of oral function and cognitive function. The level of significance was set as $\mathrm{p}<0.05$.

\section{Results}

Summary of oral conditions and cognitive function of participants are shown in Table 1. The median (Interquartile Range (IQR)) of number of teeth was 24 (16-27), maximum occlusal force was $245.5 \mathrm{~N}$ $(11 \mathrm{~N}-1090 \mathrm{~N})$, gummy jelly score was $5(0-9)$ and the average tongue pressure was $26.3 \pm 9.0 \mathrm{kPa}$. The median (IQR) of TMT A time was $46.5 \mathrm{~s}(39.0 \mathrm{~s}-53.7 \mathrm{~s})$, and TMT B time was $107.7 \mathrm{~s}$ (86.2s-131.2s).

A significant negative correlation was found between the number of teeth, and the TMT A and B times (Spearman's rank correlation coefficient; $r_{s}=-0.270$ and -0.194 respectively, $p<0.05$ ) (Figs. 1 and 2). A significant negative correlation was also found between the maximum occlusal force, and the TMT A and B times ( $\mathrm{rs}=-0.210$ and -0.300 respectively, $\mathrm{p}<0.05$ ) (Figs. 3 and 4). Additionally, a negative correlation was found between tongue pressure and the TMT A time ( $\mathrm{rs}=-0.223$, p $<0.05$ ) (Fig. 5) and between masticatory performance and the TMT B time $\left(\mathrm{r}_{\mathrm{s}}=-0.188\right)$ (Fig. 6).

Multiple linear regression analysis showed that number of teeth had significant negative associations with TMT-A (standardized partial regression coefficient $(\beta)=-0.504, p<0.05$ ), and maximum occlusal force had significant negative associations with TMT-B (standardized partial regression coefficient $(\beta)=-0.038, \mathrm{p}<0.05$ ) after controlling for age.

\section{Discussion}

Japan is rapidly becoming an aging society, with an aging rate of $27.3 \%$. Therefore, adequate dementia care needs to be provided because the number of elderly people with dementia is also increasing.

Dementia is the loss of cognitive functioning - thinking, remembering, and reasoning - and behavioral abilities to such an extent that it interferes with a person's daily life and activities. These functions include memory, language skills, visual perception, problem solving, self-management, and the ability to focus and pay attention. Some people with dementia cannot control their emotions, and their personalities may change. Dementia ranges in severity from the mildest stage, when it is just beginning to affect a person's functioning, to the most severe stage, when the person must depend completely on others for basic activities of daily living. Therefore, early detection of the decline in cognitive function and clarification of the cause of dementia are very important.

The TMT is among the most commonly used neuropsychological tests because of its sensitively to detect structural and functional brain damage ${ }^{6}$. Additionally, the MMSE is widely used to screen for cognitive decline and impairments ${ }^{7}$. The validity of the MMSE-J has been shown to have high sensitivity and specificity for dementia ${ }^{8)}$. Therefore, these tests were used to assess cognitive function in the present study.

Oral-related factors affecting dementia include a chronic inflammatory state of the periodontal tissue, irritation to the oral cavity and to the brain resulting from chewing movements, and malnutrition due to decreased oral function. This information has led to reports of a significant association between cognitive decline, oral health status, and the occurrence of dementia in numerous longitudinal and intervention studies in Japan and abroad ${ }^{7}$. Recently, some studies have reported a relationship between oral health and cognitive impairment ${ }^{9}$. In 1997, Kato et al reported a relationship between tooth loss and spatial memory deficits in 
senile rats; rats without molar teeth consuming a soft diet showed worse behave oral performance than rats with molar teeth consuming a solid diet ${ }^{10)}$. In addition, Kimura et al reported that low chewing ability was associated with lower cognitive functioning ${ }^{11}$. In contrast, some studies have reported that loss of teeth or chewing ability was not associated with cognitive impairment ${ }^{12)}$.

Herein, maximum occlusal force, number of teeth, gummy jelly score (mastication ability), and tongue pressure were found to be correlated with cognitive function. The sensory input through the trigeminal nerve to the teeth via the mechanism of chewing affects the development of dementia, as it increases the blood flow of the brain and promotes arousal of the brain. Thus, there is a possibility that activating the brain in relation to chewing capabilities may help prevent dementia. Further, there appears to be a close relationship between dementia and decreased oral function.

In this preliminary study, we confirmed associations between the TMT A and B times and maximum occlusal force, number of teeth, and tongue pressure. Among the diagnostic instruments, the TMT has been shown to be simple and useful for the early diagnosis of dementia, and thus, it was used in the present study. Additional studies using a larger number of cases and longer time should be conducted in the future to further examine the relationship between oral function and dementia.

\section{Acknowledgments}

This research was partially supported by Private University Research Branding Project 2017 and Japan Society for the Promotion of Science KAKENHI Grant Number JP18K17213, JP19K06873.

\section{Conflict of Interests}

The authors have declared that no COI exists.

\section{References}

1. Sakurai H, Hanyu H, Kanetaka H, Sato T, Shimizu S and Hirao K. Prevalence of coexisting diseases in patients with Alzheimer's disease. Geriatr Gerontol Int 10: 216-217, 2010

2. Deschaintre Y, Richard F, Leys D and Pasquier F. Treatment of vascular risk factors is associated with slower decline in Alzheimer disease. Neurology 73: 674-680, 2009

3. Ono Y, Yamamoto T, Kubo KY and Onozuka M. Occlusion and brain function mastication as a prevention of cognitive dysfunction. J Oral Rehabil 37: 624-640, 2010

4. Nokubi T, Yoshimuta Y, Nokubi F, Yasui S, Kusunoki C, Ono T, Maeda Y and Yokota K. Validity and reliability of a visual scoring method for masticatory ability using test gummy jelly. Gerodontology 30: 76-82, 2012

5. Nakajima Y, Ueno T, Kato-Kogoe N, Yamamoto K, Fukui N, Suwa Y, Kimura Y, Ito Y, Takahashi Y, Kasuya S, Kanou M, Terai H, Tano T and Ariyoshi Y. Study on suitability of grafted bone following mandibular reconstruction evaluated according to masticatory performance scores using half-potion gummy jelly. J Hard Tissue Biol 25: 427-430, 2016

6. Kokubo N, Yokoi Y, Saitoh Y, Murata M, Murao K, Takebayashi Y, Shinmei I, Yoshimoto S and Horikoshi M. A new device-aided cognitive function test, User experience-Trail Making Test (UX-TMT), sensitively detects neuropsychological performance in patients with dementia and Parkinson's disease. BMC Psychiatry 18: 220, 2018

7. Folstein MF, Folstein SE and McHugh PR. "Mini-mental state". A practiceal method for grading the cognitive state of patients for the clinician. J Psychiatr Res 12: 189-198, 1975

8. Ideno Y, Takayama M, Hayashi K, Takagi H and Sugai Y. Evaluation of a Japanese version of the Mini-Mental State Examination in elderly persons. Geriatr Gerontol Int 12: 310-316, 2012

9. Ghezzi EM and Ship JA. Dementia and oral health. Oral Surg Oral Med Oral Pathol Oral Radiol Endod 89: 2-5, 2000

10. Kato T, Usami T, Noda Y, Hasegawa M, Ueda M and Nabeshima T. The effect of the loss of molar teeth on spatiall memory and acetylcholine release from the partietal cortex in aged rats. Behav Brain Res 83: 239-242, 1997

11. Kimura Y, Ogawa H, Yoshihara A, Yamaga T, Takiguchi T, Wada T, Sakamoto R, Ishimoto Y, Fukutomi E, Chen W, Fujisawa M, Okumiya K, Otsuka K, Miyazaki H and Matsubayashi K. Evaluation of chewing ability and its relationship with activities of daily living, depression, cognitive status and food intake in the community-dwelling elderly. Geriatr Gerontol Int 13: 718-725, 2013

12. Chen X, Shuman SK, Hodges JS, Gatewood LC and Xu J. Patterns of tooth loss in older adults with and without dementia: a retrospective study based on a Minnesota cohort. J Am Geriatr Soc 58: 23002307,2010 